\title{
Variant on Manifestation of Duodenal Metastasis 26 Years after Initial Diagnosis of Primary Cutaneous Melanoma
}

\author{
Kumiko Kitajima ${ }^{a} \quad$ Armelle Bardier-Dupas $^{b}$ Sylvie Breton ${ }^{a}$ \\ Géraldine Rousseau ${ }^{\mathrm{a}}$ Jean-Michel Siksik ${ }^{\mathrm{a}}$ \\ Jean-Christophe Vaillant ${ }^{\mathrm{a}} \quad$ Laurent Hannoun $^{\mathrm{a}}$ \\ aDepartment of Digestive and Hepato-Biliary-Pancreatic Surgery, Liver \\ Transplantation Unit, and bepartment of Pathology, Assistance Publique - \\ Hôpitaux de Paris (AP-HP), Hôpital Pitié-Salpêtrière, Université Pierre et Marie \\ Curie - Paris VI, Paris, France
}

\section{Key Words}

Malignant melanoma - Metastatic duodenal tumor - Nonampullary duodenal neoplasm . Amelanotic melanoma

\begin{abstract}
Malignant duodenal neoplasms are relatively rare, and the diagnosis is often delayed because of their vague and nonspecific symptoms. We report the case of a 79-year-old female who had a medical history of malignant melanoma of the cheek that had initially been diagnosed at 53 years of age. Work-up revealed severe stenosis of the duodenum caused by a large mass with ulceration at the tip of its mucosal surface. Tumor biopsy led to a histological diagnosis of extremely poorly differentiated carcinoma, but it was impossible to determine whether the lesion was a primary neoplasm or represented secondary involvement. Pancreatoduodenectomy was performed, and the surgical specimen showed a protuberant tumor in the nonampullary region of the second portion of the duodenum. Final diagnosis of metastatic duodenal melanoma was made by immunohistological examination. She is currently alive without recurrence 28 months after the surgical treatment.
\end{abstract}

\section{Introduction}

Malignant duodenal neoplasms are relatively rare, and the most common site of occurrence is the periampullary region. Due to nonspecific symptoms, the diagnosis is 
frequently delayed unless acute complications such as obstruction, massive bleeding or perforation develop. We report a case who complained of weakness only. Endoscopy revealed a fairly large duodenal mass in the nonampullary region of the second portion of the duodenum, but the pathological diagnosis could not be made before surgery, even after histological examination of the endoscopic biopsy.

\section{Case Report}

A 79-year-old woman was admitted to the Department of Cardiovascular Surgery complaining of weakness. She had undergone aortic valve replacement surgery three months before because of severe aortic valve stenosis. Prior to the aortic valve replacement procedure, esophagogastroduodenoscopy had been performed because of the presence of microcytic anemia (hemoglobin level $10.8 \mathrm{~g} / \mathrm{dl}$; normal range $12-16 \mathrm{~g} / \mathrm{dl}$ ), and it had shown entirely normal findings (fig. 1a). When the patient was readmitted, the anemia had markedly progressed (hemoglobin level $8.8 \mathrm{~g} / \mathrm{dl}$ ). Duodenoscopy revealed a severe stenosis and ulceration at the tip in the second portion of the duodenum despite the absence of any digestive complaints. The mucosal surface was highly friable and bled easily (fig. 1b). Biopsy specimens of the lesion revealed extremely poorly differentiated carcinoma, and it was impossible to determine whether the lesion was primary or metastatic. Computed tomography showed circumferential but asymmetric stenosis due to severe parietal thickening in the second portion of the duodenum (fig. 2). This parietal thickening extended vertically and measured $26 \times 60 \mathrm{~mm}$. Although several periduodenal lymph nodes were detected, they were all less than $10 \mathrm{~mm}$ in diameter. No other lesions, including dilatation of the main pancreatic duct or biliary system, were found, and a primary duodenal cancer was suspected based on the above findings. Laboratory studies, including serum CA19-9 and CEA, did not yield any abnormal findings except a slight elevation of the LDH level (456 U/l; normal range 190-390 U/l), and the patient was referred to our institution. The patient had a history of malignant melanoma (MM) in the left cheek diagnosed at 53 years of age, and she had undergone local surgical excision six times for relapses over a 10-year period. The subtype of MM diagnosed initially was lentigo maligna. She later underwent left lower pulmonary lobectomy for a rapidly growing solitary mass at 71 years of age. However, no details regarding the diagnosis of the pulmonary tumor were available when she was admitted to our institution.

At laparotomy, a large, hard tumor was palpable in the second portion of the duodenum, but there were no metastatic lesions in other organs, ascites, or intraabdominal seedings. Pancreatoduodenectomy with en bloc resection of the periduodenal lymph nodes was performed. The resected specimen contained a solid mass with a maximum diameter of $5.5 \mathrm{~cm}$ in the second portion of the duodenum, and the duodenal papilla was completely intact. Histological examination showed a poorly differentiated tumor composed of epithelioid cells. The tumor was limited to the submucosal layer and muscularis propria and was covered by normal mucosa with no evidence of adenomatous duodenal proliferation (fig. $3 \mathrm{a})$. There was cytonuclear atypia with numerous huge nuclei and many mitoses (fig. 3b). Immunohistochemical staining of the tumor was strongly positive for HMB45 and Melan-A (fig. 3c, d). Tumor cells were negative for epithelial immunochemical markers (cytokeratins, AE-1, AE-3, EMA, KL-1) and for common leukocyte antigen (CD45). Consequently, the duodenal tumor was confirmed to be metastatic MM. The histological findings in the lung resected 8 years before were investigated by requesting sections from the hospital where the resection had been performed, and the findings of the duodenal tumor were found to closely resemble the histopathology of the resected lung. The patient is alive and recurrence-free 28 months after the operation.

\section{Discussion}

Duodenal tumors occupy nearly one third of all gastrointestinal neoplasms, but their incidence is uncommon. Primary malignant neoplasms of the duodenum account for about only $0.3 \%$ of all malignancies of the gastrointestinal tract $[1,2]$. Regarding secondary involvement, autopsy studies have shown that metastases of any type to the small bowel account for only $1-2 \%$ of all metastases [3]. It is difficult to know the true incidence of metastatic neoplasms in the duodenum because the duodenal metastasis is concealed in most studies of small intestinal secondaries as mentioned above, and it has been mainly published as single case reports. Although the most common site of 
occurrence of duodenal tumors is the periampullary region, the duodenal neoplasm in our patient was located in the nonampullary region of the second portion of the duodenum, and the diagnosis of metastatic melanoma was only established by immunohistochemical study of the surgical specimen.

MM arises from pigment cells in the skin and is well known to be an aggressive and lethal malignancy. Although only a small percentage of cutaneous malignancies are due to $\mathrm{MM}$, it accounts for the vast majority of skin cancer-related deaths. Despite the fact that the primary melanoma lesion is completely excised in most cases, metastasis occurs in about one third of patients [4], and melanomas have a tendency to metastasize to any part of organs and sometimes metastasize long after diagnosis of the primary lesion. Their unpredictable behavior is the most exasperating feature of this malignancy.

MM is the most common extraabdominal primary neoplasm to metastasize to the gastrointestinal tract, including the duodenum [5]. The abundant blood supply of the intestine may account for the predilection of melanoma to metastasize to the small intestine, but the correct diagnosis is seldom made preoperatively. There is a large discrepancy between the incidence of metastatic melanoma of the upper gastrointestinal tract reported pre and post mortem (4-8\% vs. $40-60 \%)$ by several investigators $[5,6]$. Capizzi and Donohue reported that $80 \%$ of duodenal metastases by melanoma were first diagnosed at surgery [7]. These findings seem to illustrate well the difficulty of diagnosing intestinal metastasis of melanoma. In most cases, the primary neoplasm has already been identified by the time the duodenal tumor is biopsied, and it helps to make the histological diagnosis of secondary involvement. Although our patient had a history of cutaneous MM, the nonpigmented duodenal neoplasm discovered 26 years after the diagnosis of MM did not immediately suggest a diagnosis of metastatic MM.

Endoscopic examination of the duodenal neoplasm reveals a protuberant submucosal tumor, with ulceration at the tip as the lesion grows larger in many cases, and black pigmentation in typical metastatic melanoma. However, metastatic melanoma was not diagnosed preoperatively in our case despite histological examination with biopsy, and the diagnosis was established only by immunostaining of the surgical specimen. The complete lack of pigmentation and the impossibility of aggressive biopsy because of the strong tendency of the tumor to bleed uncontrollably and its fragility are suggested as reasons for the delay in diagnosis. The absence of pigmentation in a tumor can lead to misdiagnosis for MM. However, pigmentation is not a specific feature of metastases of $\mathrm{MM}$ even when the primary lesion is pigmented, and distant metastases tend to be less pigmented than the primary tumor $[8,9]$. Although amelanosis is likely to be associated with multiple changes or abnormalities in the gene expression that results in a single phenotype, the precise underlying mechanism of amelanosis has not been determined. As has been described in several reports, discoloration in melanomas represents a change from melanocytes to poorly differentiated cells that no longer produce melanin and indicates rapid growth of the tumor $[10,11]$.

Immunohistochemical study is helpful in identifying metastases of melanoma, and in our case, immunostaining with HMB45 and Melan-A was conclusive in making the diagnosis of metastatic duodenal melanoma. However, no specific antibody is available to identify MM. A combination of antibodies should therefore be used to make an accurate diagnosis of melanoma.

The virulence of MM in its metastatic pattern is quite evident by the diffuse involvement in multiple organ systems. Cutaneous melanoma metastasizes by several pathways, and one third of melanomas metastasize to distant sites without first 
metastasizing to any intermediate sites. In our patient, isolated pulmonary metastasis and duodenal metastasis occurred at different times, 18 and 26 years, respectively, after the initial diagnosis of cutaneous melanoma. To the best of our knowledge, the distant metastatic melanoma 26 years after the diagnosis of primary cutaneous lesion is the most delayed incidence as a detailed case report.

Although there were long periods of tumor cell dormancy before the metastasis emerged in either organ, melanoma cells may have spread via blood vessels during repeated surgical treatment for relapses. The subtype of the MM in our patient was lentigo maligna, also known as 'mélanome de Dubreuil', which is a precursor lesion of lentigo maligna melanoma $[12,13]$. Lentigo maligna melanoma is an invasive type of lentigo maligna that progresses by growing vertically beyond the dermal-epidermal junction. Lentigo maligna is known to have a strong tendency to repeatedly recur as in our case. Most recurrences do not pose an immediate threat to the patient's life, but residual lesions as a result of inadequate treatment or atypical melanocytes that are resistant to destructive therapy may develop into invasive melanoma during the course of repeated excisions. In our case the initial lesion seems to have become an invasive melanoma and undergone gradual changes in features during the course of repeated surgical treatment. MM can give rise to distant metastases any time after the initial diagnosis of the primary lesion irrespective of previous sites of metastasis.

Patients with stage IV melanoma have a very poor prognosis, and only a few of them survive beyond one year (median survival 4 to 9 months [14]). Although systemic therapeutic strategies, including chemotherapy and immunotherapy, have been developed, they have only succeeded in delaying the development of metastasis in a few cases, and there has been no proof of significant prolongation of survival. Surgery for stage IV melanoma is also considered palliative treatment for salvage from life-threatening complications. However, a considerable number of studies have shown a significant impact of surgical interventions on the survival rate in selected cases [15].

Several investigators have identified predictors of survival in patients with distant metastasis by melanoma: (1) location of the metastasis, (2) number of metastatic lesions, (3) disease-free interval since initial treatment of the primary lesion, and (4) serum lactate dehydrogenase level $[4,16]$. Despite recurrence of MM six times at the primary cutaneous site in our case, the pulmonary and duodenal metastases occurred in isolation, and there were long disease-free intervals before metastasis to both organs. These favorable conditions appear to have led to the long survival time of our patient and support aggressive surgical management, and we have concluded that complete resection can lead to cure of this fatal malignancy in certain cases. In fact, the ability to perform a complete resection is the only treatment-related prognostic factor. Surgery should be performed to treat visceral metastasis not only for salvage from life-threatening conditions, such as intestinal obstruction, but to achieve a complete cure under certain conditions.

In conclusion, it should be borne in mind that distant metastasis of MM can occur at any time, even long after the diagnosis of the primary lesion. Furthermore, it is important to note that metastases of melanomas tend to become amelanotic. When only one organ is involved by metastasis and complete surgical resection of the metastatic lesion is possible, aggressive surgical resection should remain a viable treatment option. Complete resection is very likely to provide long-term disease-free survival in selected cases. 
Fig. 1. Endoscopic view of the duodenum. a No lesion was detected 3 months before. b Protuberance with smooth surface resulted in severe stenosis in the descending duodenum.
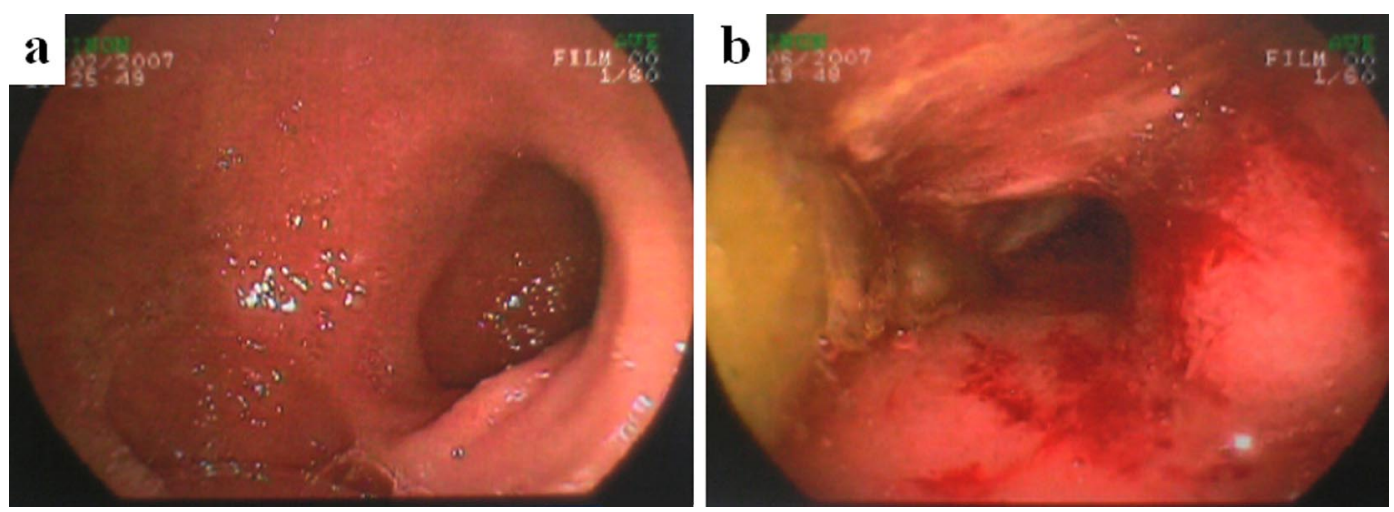

Fig. 2. Abdominal computed tomography scan showing a large mass measuring $26 \times 60 \mathrm{~mm}$, extending vertically in the second portion of the duodenum. The protrusion into the lumen caused severe narrowing of the duodenal lumen.
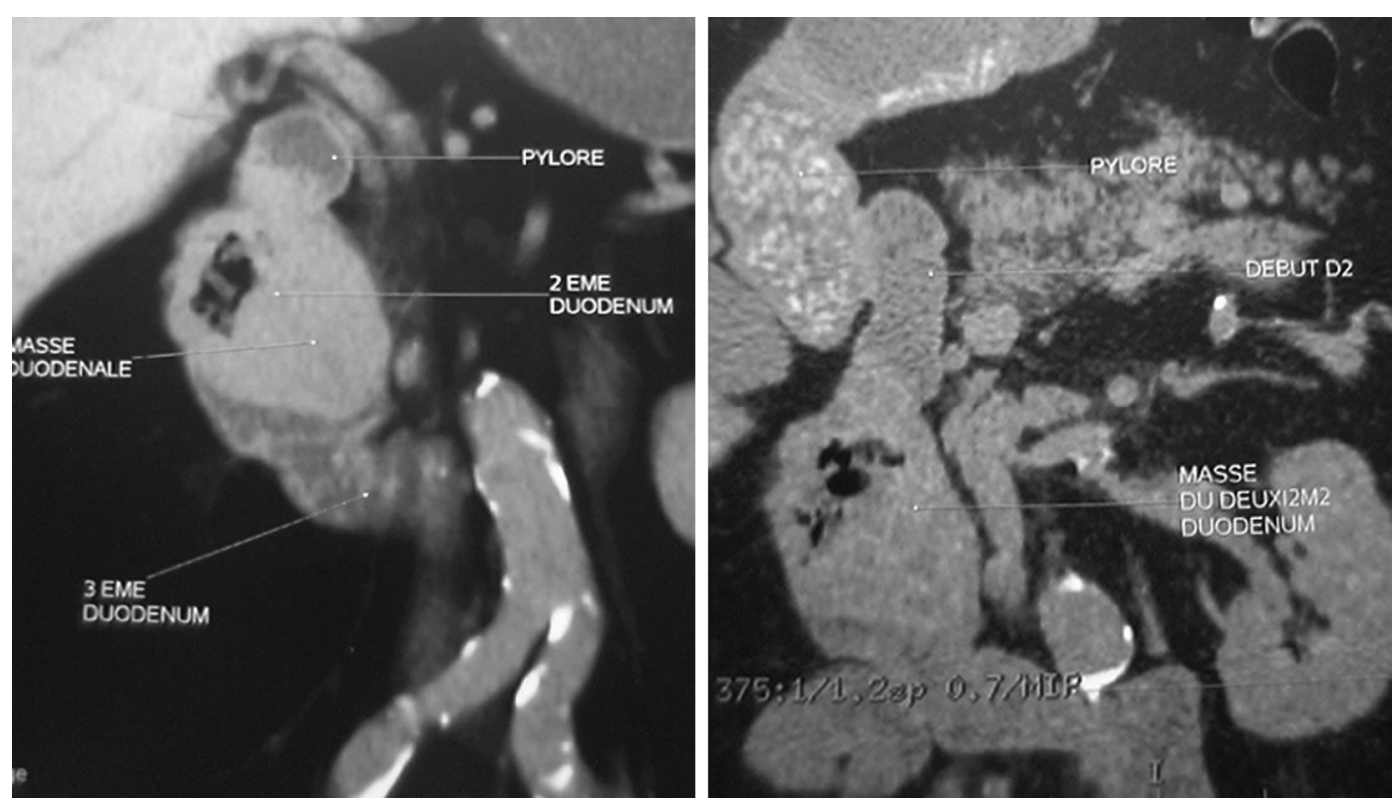


\begin{tabular}{r|l|l|l}
$\begin{array}{r}\text { Case Reports in } \\
\text { Gastroenterology }\end{array}$ & $\begin{array}{l}\text { Case Rep Gastroenterol 2010;4:93-99 } \\
\text { D0I: 10.1159/000290383 }\end{array}$ & Published online: March 17, 2010 & $\begin{array}{l}\text { O 2010 S. Karger AG, Basel } \\
\text { ISSN 1662-0631 } \\
\text { www.karger.com/crg }\end{array}$ \\
\hline
\end{tabular}

Fig. 3. Photomicrograph of a histological section of the resected duodenal specimen. a Proliferation of tumor cells in the submucosal layer under an intact duodenal mucosa $(\mathrm{H} \& \mathrm{E}$, original magnification $\times 40$ ). $\mathbf{b}$ The tumor exhibited an epithelioid pattern with large polygonal nonadherent cells containing abundant nonpigmented cytoplasms, and nuclei containing large nucleoli, and there were numerous mitoses (H\&E, original magnification $\times 200)$. c Melanoma cells that stained positive immunohistochemically (HMB45, original magnification $\times 400)$. d Melanoma cells that stained positive for Melan-A (Melan-A, original magnification $\times 400$ ).

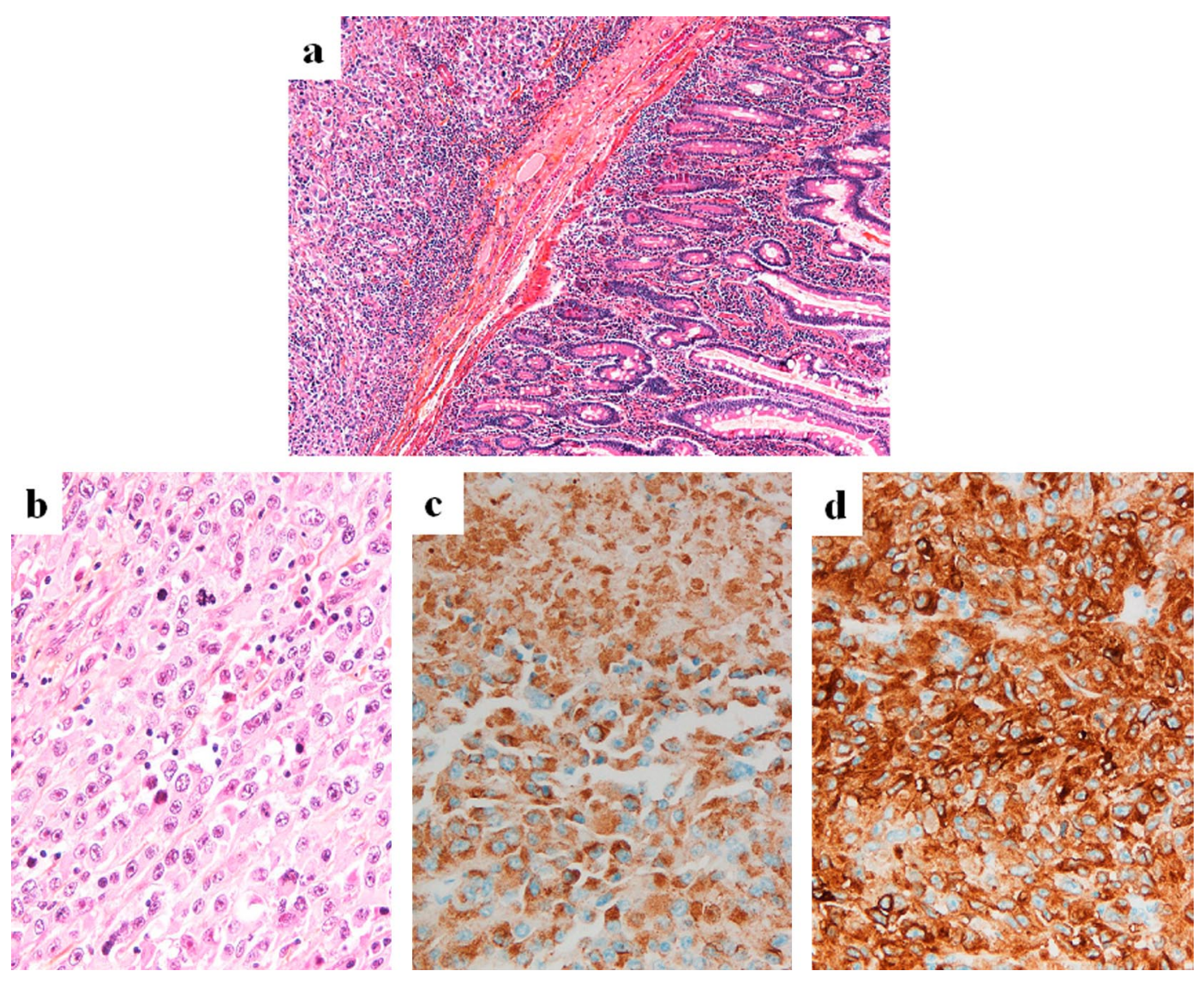




\section{References}

1 Farah MC, Jafri SZ, Schwab RE, et al: Duodenal neoplasms: Role of CT. Radiology 1987;162:839-843.

2 Alwmark A, Anderson A, Lasson A: Primary carcinoma of the duodenum. Ann Surg 1980;191:13-18.

3 Adamo R, Greaney PJ Jr, Witkiewicz A, Kennedy EP, Yeo CJ: Renal cell carcinoma metastatic to the duodenum: Treatment by classic pancreaticoduodenectomy and review of the literature. J Gastroint Surg 2008;12:1465-1468.

-4 Spanknebel K, Kaufman HL: Surgical treatment of stage IV melanoma. Clin Dermatol 2004;22:240-250.

5 Kadakia SC, Parker A, Canales L: Metastatic tumors of the upper gastrointestinal tract: endoscopic experience. Am J Gastroenterol 1992;87:1418-1423.

-6 Das Gupta T, Brasfield R: Metastatic melanoma: a clinicopathologic study. Cancer 1964;17:1323-1327.

7 Capizzi PJ, Donohue JH: Metastatic melanoma of the gastrointestinal tract: a review of the literature. Compr Ther 1994;20:20-23.

$\rightarrow 8$ Koch SE, Lange JR: Amelanotic melanoma: the great masquerader. J Am Acad Dermatol 2000;42:731-734.

-9 Smith SH, Goldschmidt MH, McManus PM: A comparative review of melanocytic neoplasms. Vet Pathol 2002;39:651-678.

10 Hashimoto A, Aoki M, Kawana S: Two cases of amelanotic malignant melanoma. Nishinihon JDermatol 2002;64:45-47.

-11 Lazarević V, Tiodorović J, Tiodorović-Živković D, Stanojević M, Popović D, Janković A: Immunophenotyping of amelanotic melanoma. A case report. Acta Dermatovenerol Alp Panonica Adriat 2006;15:141-143.

$\checkmark 12$ Cohen LM: Lentigo maligna and lentigo maligna melanoma. J Am Acad Dermatol 1995;33:923-936.

13 Marko L: Current clinical overview of cutaneous melanoma. Br J Nurs 2008;17:300-305.

14 Balch CM, Soong SJ, Gershenwald JE, et al: Prognostic factors analysis of 17,600 melanoma patients: validation of the American Joint Committee on Cancer melanoma staging system. J Clin Oncol 2001;19:3622-3634.

15 Ollila DW, Essner R, Wanek LA, Morton DL: Surgical resection for melanoma metastatic to the gastrointestinal tract. Arch Surg 1996;131:975-979.

16 Allen PJ, Coit DG: The surgical management of metastatic melanoma. Ann Surg Oncol 2002;9:762-770. 